\section{SCIENCE CHINA \\ Physics, Mechanics \& Astronomy}

-Editorial•

Editor's Focus
November 2021 Vol. 64 No. 11: 111061

https://doi.org/10.1007/s11433-021-1765-8

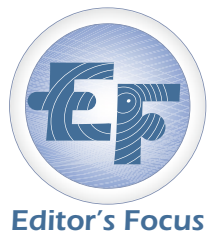

\title{
New connection between dark matter direct detections, astrophysical and cosmological observations with self-interacting dark matter
}

The self-interacting dark matter (SIDM) model is an ideal candidate for explaining the discrepancy between small-scale structure observations and predictions by the prevailing collisionless cold dark matter (CDM) model. SIDM indicates the existence of a light mediator with a typical mass of $10 \mathrm{MeV}$. Searching for SIDM particles has therefore become one important alternative to the traditional weakly interacting massive particles (WIMPs) in direct detection experiments such as PandaX. Of note, the constraints obtained from direct detections are associated with the unknown mixing parameter (such as photon kinetic mixing) that describes the strength of the coupling between the mediator and ordinary particles. As previously reported, this makes it difficult to directly compare the direct detection constraints with the astrophysical constraints on SIDM models [1].

Recently, a team led by Prof. Yong Yang at Shanghai Jiao Tong University and Prof. Hai-Bo Yu [2] at the University of California, Riverside, proposed a new way to connect the results from direct detection experiments, astrophysical observations and cosmological observations. The null SIDM signals obtained in direct detection experiments set limits on the dark matter (DM) mass, mediator mass and mixing parameter. The astrophysical observations put constraints on DM vs. mediator mass. The standard Big Bang Nucleosynthesis (BBN) scenario successfully predicts light element abundances, which might be changed if the mediator decays to ordinary particles via the kinetic mixing portal.

Using the constraints derived from the full DM dataset of PandaX-II experiment, the favored DM vs. mediator mass region by observations of DM halos from dwarf galaxies to galaxy clusters [3], and the benchmark BBN constraints on mediator lifetime [4], they showed that, overall, the dark sector needs to be colder than the visible sector in the early universe for the DM masses ranging from about 10 to $200 \mathrm{GeV}$. This work also demonstrates that direct detections, astrophysical and cosmological observations provide complementary tools for probing the self-interacting nature of DM particles.

YiPeng Jing

School of Physics and Astronomy, Shanghai Jiao Tong University, Shanghai 200240, China

1 X. Ren, L. Zhao, A. Abdukerim, X. Chen, Y. Chen, X. Cui, D. Fang, C. Fu, K. Giboni, F. Giuliani, L. Gu, X. Guo, K. Han, C. He, D. Huang, S. He, X. Huang, Z. Huang, X. Ji, Y. Ju, Y. Li, H. Lin, H. Liu, J. Liu, Y. Ma, Y. Mao, K. Ni, J. Ning, A. Tan, H. Wang, M. Wang, Q. Wang, S. Wang, X. Wang, S. Wu, J. Xia, M. Xiao, P. Xie, B. Yan, J. Yang, Y. Yang, H. B. Yu, J. Yue, T. Zhang, J. Zhou, N. Zhou, Q. Zheng, and X. Zhou, Phys. Rev. Lett. 121, 021304 (2018), arXiv:
1802.06912 .

2 J. Yang, et al. (PandaX-II Collaboration), Sci. China-Phys. Mech. Astron. 64, 111062 (2021).

3 R. Huo, M. Kaplinghat, Z. Pan, and H. B. Yu, Phys. Lett. B 783, 76 (2018), arXiv: 1709.09717.

4 P. F. Depta, M. Hufnagel, and K. Schmidt-Hoberg, J. Cosmol. Astropart. Phys. 2021, 011 (2021), arXiv: 2011.06519. 